\title{
A technique for accurate identification of alpha frequency in the EEG.
}

\author{
RONALD S. HOSEK and ARTHUR S. WILSON \\ Departments of Neurosurgery and Psychiatry \\ Medical College of Wisconsin and Research Service \\ VA Center, Wood (Milwaukee), Wisconsin 53193
}

An economical and highly accurate electronic filter for the detection of narrow frequency bands in the electroencephalogram (EEG) is described. The apparatus employs solid state integrated circuits and digital logic to detect and provide feedback output. In the example given, this device acts as an alpha rhythm discriminator and provides the accuracy of a more expensive computer simulated filter.

The present literature in both popular and scientific journals is replete with articles dealing with the current vogue in autocontrol of cortical electrical activity. The general approach involves the use of a variety of instruments to record and filter the electrial signals from scalp electrodes in order to extract the alpha rhythm. Once indentified, attempts are made to modify the duration of the alpha frequencies by use of behavioral feedback techniques (Nowlis \& Kamiya, 1970). The resultant control of the alpha rhythm is thought to be clinically therapeutic (Green, Green, \& Walters, 1970) and may also be indicative of an altered state of consciousness (Kamiya, 1968, 1969).

Present techniques utilize tuned active or passive filters to discriminate the alpha rhythm from the background electrocortical activity or, in some instances, may utilize on-line control with a digital computer (Boudrot, 1972). While the latter procedure may permit accurate isolation of the alpha bandpass, it involves a rather extensive investment in apparatus. The former methods using tuned filters, although inexpensive, suffer from a lack of sufficient selectivity for scientifically vigorous investigations. This paper describes a filter that combines component economy with the accuracy of a high-powered computer-simulated filter.

The alpha rhythm discriminator to be described takes advantage of the temporal properties of the alpha rhythm range. Since the alpha rhythm occupies a frequency range of $8.12 \mathrm{~Hz}$, the implication is that one may define an alpha period range of $1 / 2$ to $1 / 12 \mathrm{sec}$. Therefore, if scalp activity is squared, logic circuitry can be assembled to identify activity that falls within the alpha period range on a cycle-by-cycle basis. For each cycle falling within the desired range, the apparatus will produce a pulse. The resulting pulse train that is produced as the result of prolonged alpha periods may be integrated to produce a constant output.

The working of the device may be more easily understood by examining Fig. 1. Prior to being fed into the filter, the ongoing complex EEG signal is amplified and clipped (i.e., squared) to convert it into a square pulse train. Then durations between axis crossing of this pulse train correspond exactly to the durations between axis crossing of the original signal, thus preserving the major frequency information. When an arbitrary pulse arrives at the filter input, its leading edge (corresponding to positive-going axis crossing) is sensed, thus activating or enabling the high and low timing circuits (Fig. 2). These timing circuits are preset to provide pulses at a latency of from 42 to $62.5 \mathrm{msec}$, corresponding to half the alpha period range. When the trailing edge (i.e., negative-going axis crossing) of the incoming pulse appears, a very rapid pulse is generated through a logical decision tree. This decision tree provides a true output if the input falls with the true window and no output if the signal falls outside the true window. The trailing

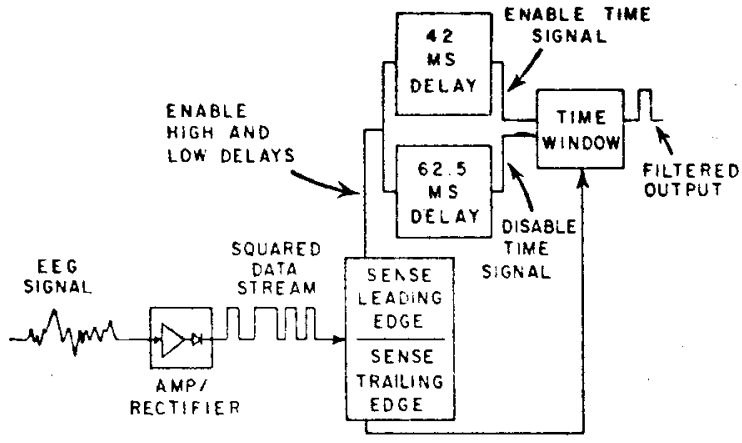

Fig. 1. Block diagram of filter logic. The incoming data stream is squared, and the leading edges of the pulses in the resultant pulse train serve to enable the logical true window. If the trailing edge of the pulse falls within the window, the unit provides an output pulse (see Fig. 2).

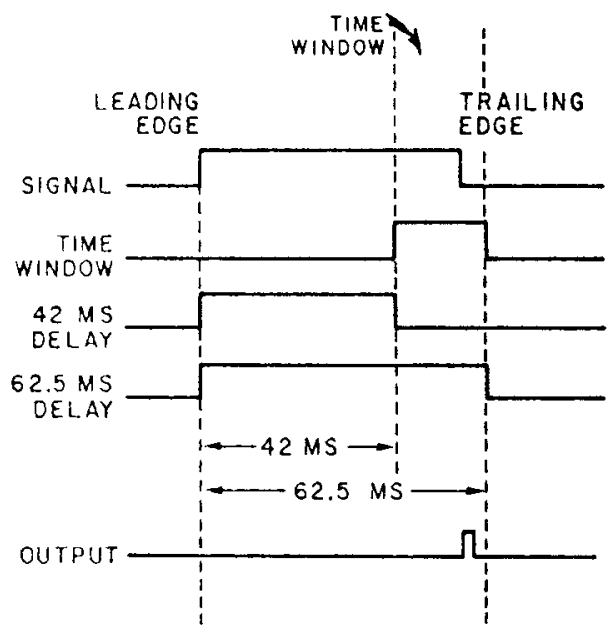

Fig. 2. Timing diagram of fil ter logic. 


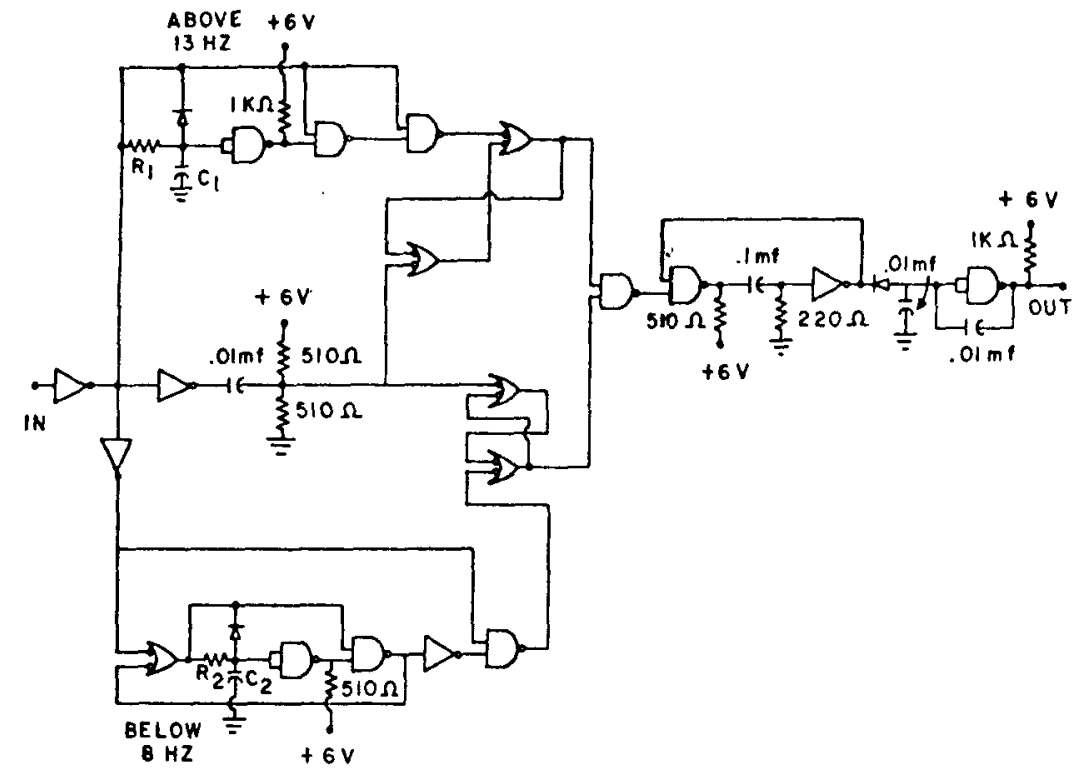

Fig. 3. Circuit diagram of filter. Commercially available integrated logic circuits are used throughout. The $\mathrm{R}_{1} \mathrm{C}_{1}$ time constant determines the upper edge of the passband while $R_{2}$ and $C_{2}$ determine the lower edge. edge pulse also serves as a reset signal to enable the logic tune window to deal with the next leading edge of the ongoing data signal.

The circuitry required to perform these functions is electronically elegant, yet simple to assemble in that it utilizes integrated circuit logic elements (Fig. 3). Using commercially available components, the circuit may be assembled for less than $\$ 20.00$.

While the unit described is for filtering frequencies in the $8.12 \mathrm{~Hz}$ range, the identical logic can be utilized to produce similar filters for other biologically important frequencies in the EEG by choosing the values of $R_{1}$, $C_{1}, R_{2}$, and $C_{2}$ to obtain the desired time constants.

\section{REFERENCES}

Boudrot, R. An alpha detection of feedback control system Psychophysiology, 1972, 9, 461-466.

Green, E. E., Green, A. M., \& Walters, E. D. Self-recognition of internal states. In $\mathrm{J}$. Rose (Ed.), Proceedings of the international congress of cybernetics. London: Gordon \& Breach, 1970.

Kamiya, J. Conscious control of brain waves. Psychology Today, $1968,1,57-60$.

Kamiya, J. Operant control of the EEG alpha rhy thm and some of its reported effects on consciousness. In C. T. Tart (Ed.), Altered states of consciousness. New Y ork: Wiley, 1969.

Nowlis, D. P \& Kamiya, J. The control of electroencephalographic alpha rhythms through auditory feedback and the associated mental activity. Psychophysiology, 1970, 6, 476-484.

(Received for publication November 25, 1972; revision received April 20, 1973.) 